\title{
Child Victims of U.S. Motor Vehicle Crashes: Time from Crash to Hospital Arrival Among Cases Documented in the 2003 Fatality Analysis Reporting System (FARS) Database
}

Mahoning County District Board of Health

50 Westchester Drive Yougstown, $\mathrm{OH} 44118$, U.S.A. Telephone: $(1+330)$ 270-2855 Fax: $(1+330) 270-9194$ e-mail: Ifrisch@neoucom.edu

\section{LARRY FRISCH}

\begin{abstract}
There have been recent proposals to enhance organization and funding of emergency care in the U.S. because of perceived deficiencies - especially in services for children. Motor vehicle crashes are among the leading causes of death and disability for both adults and children in the United States and place a heavy burden on emergency and trauma care. This study uses the Fatality Accident Reporting System (FARS) to examine the length of time between vehicle crash and hospital arrival for crashes involving seriously injured children transported to hospital in 2003. Only about 1/3 (1868) of 5436 crashes had data for hospital arrival time. For those cases that could be evaluated, median transport time was 46 minutes (52 minutes for rural crashes, 35 minutes for those in urban areas). There was substantial delay in a minority of crashes. Delays occurred both between crash and emergency services (EMS) notification and between EMS contact and hospital arrival and were more common in rural crashes. Other research has shown that prolonged transport times are associated with worsened trauma outcomes. Since states vary in the extent and sophistication of their trauma services we sought, but failed to find, correlation between number of trauma centers (Level I and II or all-level) per million population and median arrival time calculated for each U.S. state. Integrated trauma care begins at the site of injury and continues through posthospital care. Time between injury and hospital admission is one important indicator of trauma system performance.
\end{abstract}

Keywords: Accidents, Traffic; Emergency Medical Services; Children; Transportation of Patients; Trauma Centers; Injuries

\section{Introduction}

In June, 2006 the U.S. Institute of Medicine released three reports on the state of the country's emergency treatment. One of these reports paints a particularly problematic picture of emergency care for children (1). Only half of emergency departments in U.S. hospitals have more than $85 \%$ of recommended supplies to handle pediatric emergencies, and only $6 \%$ of hospitals have all of the recommended supplies. Many hospitals lack the ability to handle pediatric trauma, but only half of these have organized systems for transferring these cases to an appropriate trauma center. There is a deficit of continuing education in pediatric emergency care for trauma personnel. As a result, evidence suggests that children may not be properly stabilized prior to transfer, and compared to adults, children may receive suboptimal care. While problems are significant in urban areas, they may be more severe in rural areas where specialized services of any sort are often in short supply. Many of these deficiencies have been addressed, at least to some extent, by grants provided through the federal Emergency Medical Services for Children program, a program slated for elimination in the current national budget proposed by President Bush. The Institute of Medicine has recommended increasing the current appropriation for this program to $\$ 37.5$ million yearly, a tiny fraction of the total $\$ 1.9$ trillion U.S. health expenditures.

The importance of pediatric emergency and intensive care is nowhere more evident than in motor vehicle trauma. Motor vehicle crashes are the lead- 
ing cause of death for U.S. children between the ages of 2 and 14, and children in this age group comprise 5\% of all motor vehicle deaths. They also account for $9 \%$ of all injuries in motor vehicle crashes. While absolute numbers of fatalities have been decreasing in the past decade, probably due primarily to better use of child restraints and increasing enforcement of seat belt laws, there are still over 2000 deaths and over 250,000 injuries among children in the United States. Most of these injuries are cared for in the emergency medical system cited as deficient in the Institute of Medicine report.

In recent years, there have been increasing efforts to create trauma systems in U.S. states in an effort to assure the smooth transfer of injured victims into centers that are equipped and staffed to provide optimal care. In 2002, the American Trauma Society described parameters for a national trauma system that included injury prevention, pre-hospital care, acute care, and post-hospital care (2). Among the recommendations of this report are for coordinated care using standardized protocols, strategically-placed transport vehicles, a national system for responding to emergency telephone calls, enhanced communication systems. According to this report facilities for reception of trauma must be distributed appropriately and capable of handling multiple casualties at the same time. Communications and database systems must be in place to ensure a match of trauma to the availability of appropriate care, and the respective roles of all facilities providing emergency care will be defined.

Partly in response to the Trauma Society's document, MacKenzie et al performed a national assessment of U.S. trauma centers (3). While their focus was not on children (centers providing care only to children were excluded from the analysis), the authors found many deficiencies in states' trauma organization. Data was derived from interviews with directors of trauma centers and from national data sources. Of the 50 U.S. states, 35 had formal trauma systems. Even in the states with trauma systems, less than 1/3 of Level I and II trauma centers were "verified" by the American College of Surgeons. Level I centers provide the highest level of definitive care for trauma. The study identified 190 Level I centers of which only 48 were ACS-verified. States varied widely in the number of designated trauma centers, and this variability was only somewhat related to relative population. The number of level I and II centers per million population ranged from 0.19 (Arizona) to 7.8 (North Dakota). Hospitals designated as trauma centers were more likely to be private, larger, and in urban areas. These hospitals were also more likely than nontrauma centers to have post-graduate (residency) training programs.

Automobile-related trauma is a major source of admissions to trauma centers at all levels. The U.S. National Highway Traffic Safety Agency sponsors the Fatality Accident Reporting System (FARS) (4) which details all motor vehicle crashes that result in at least a single fatality. FARS allows computer access to data from 1994-2004 and is a major source for U.S. data on fatal motor vehicle crashes. $(4,5)$ The purpose of the present study was to determine, to the extent possible, time from crash to arrival at a hospital for children seriously injured in motor vehicle crashes. While rapid transport is only one aspect of a well-functioning trauma system, it is both important for optimal care and potentially accessible to unambiguous measurement. A secondary purpose was to explore any correlation of crash to hospital time in individual states with the number of trauma centers per million state inhabitants.

\section{Methods}

Data was downloaded into Microsoft Excel from the FARS computer database on all fatal U.S. crashes occurring in 2003 in which at least one victim was under the age of 19, at least one seriously injured victim was transported to a hospital, and for which data on time of crash and time of hospital arrival were available. "Serious injury" was defined as a coding of either death or "incapacitating injury" in the FARS database. Urban and rural classes were defined using entries in the FARS "Roadway Function Class" field. Fars records time data for four events in a crash: crash time, notification of the emergency management system, on-site arrival of emergency medical services (EMS), and victim arrival at the initial hospital. 2003 information on individual state trauma systems, including number of level I and Il centers per million population were taken from the study by MacKenzie et al (3) and statistical analyses were performed using SPSS version 12.0.1.

\section{Results}

In 2003 there were 5436 U.S. crashes in which at least one victim was under the age of 19. Since FARS allows cases to be filed in the absence of data in most time fields, complete time information was available for only about one third (1868) of these crashes. Only this partial subset of crashes was analyzed in the present study. 2541 persons under age 19 were killed or severely injured in these crashes. Nearly all cases from ten states and the District of Columbia (California, Florida, Illinois, Maryland, New Hampshire, New Jersey, Michigan, New Mexico, South Carolina, and Tennessee) had missing data for the hour of hospital arrival. These eleven jurisdictions accounted for 1404 of the 3568 incompletely documented crashes; the remaining 2166 were distributed among other states.

In those cases where time could be established, the median elapsed time between crash and hospital arrival was 46 minutes (range 0 to 900 minutes). Median time from crash to hospital for rural crashes was 52 minutes compared to 35 minutes for crashes occurring on urban roadways. There was generally very little delay between crash time and contact with the emergency medical system. Median time between crash and EMS contact was 3 minutes with a range of 0 minutes to about 10 hours. Of crashes in the top (prolonged) con- 
tact decile, 90\% occurred in rural areas, a disproportionate proportion, given that overall, only about 2/3 of crashes in this sample were rural. The median EMS arrival time for all crashes after contact was made was 9 minutes. The median EMS arrival time for rural areas (11 minutes) was only modestly higher than that for urban areas (6 minutes). In 91 crashes there was a delay of 30 minutes or more from EMS notification to arrival, and $90 \%$ of these delayed ambulance arrivals occurred in rural areas.

For ambulance arrivals in less than the median time, the proportion of victims who eventually expired was higher in both urban and rural areas. For example, in urban areas, the mortality proportion was 58\% when EMS arrived in less than the 6 minute median time, whereas mortality for victims was only $46 \%$ when EMS arrival exceeded the 6 minute median. The same pattern was seen in rural areas, though the difference was less marked (36\% mortality for arrival times below the 11 minute median, 43\% for times greater than the median.)

Since states with a higher density of trauma services might have shorter crash-to-hospital arrival times we examined this relationship using correlation analysis. There was no significant correlation between median hospital arrival time and density of trauma services per million inhabitants. Mean hospital arrival time was positively correlated with the number of trauma centers (all levels) per million population (Spearman's rho $=0.482, p=.011$ ). There was a non-significant correlation (rho $=$ .385, $p=0.069$ ) between mean arrival time and number of trauma centers per million population when analysis was restricted to level I and II centers only.

\section{Discussion}

Survival after trauma is determined by many factors including extent of initial injury, rapidity of emergency response, quality and speed of initial stabilization, definitive surgical care - when indicated, and post-surgical intensive care - when needed. This study looked at the rapid- ity of emergency response and transfer to hospital among children seriously injured in vehicle crashes in the United States in 2003. Median time from crash to hospital arrival differed by only 17 minutes difference between rural and urban areas, however the median arrival time of 46 minutes was probably longer than might be optimal. In most crashes EMS notification was rapid, and emergency personnel were on the scene in a median of six minutes (urban) to eleven minutes (rural). In some cases there were lengthy delays between crash and EMS contact or between contact and arrival. These delays occurred disproportionately in rural areas but were noted in urban areas as well. FARS data does not document whether either extrication or stabilization were required, but either could have prolonged crash-site care. In addition, a significant proportion of seriously injured persons (16\%) were in crashes with multiple victims that may have required additional time for pre-transport care. Perhaps surprisingly, victims who arrived at hospital in less than the median time had a higher mortality experience than those whose transport was delayed. There are several potential explanations for these findings. More severely injured patients - who would be expected to have a higher mortality rate - may have been treated with a "scoop and run" triage policy emphasizing rapid transport over more lengthy stabilization given to the less critically injured, or alternatively less time taken for stabilization at the scene may itself result in higher subsequent mortality. There is data showing that delayed transport to definitive care is associated with higher mortality (7), though stabilization in accord with American College of Surgeons guidelines - at least in a hospital setting - clearly improves survival (8).

Although the arguments for organized state-wide trauma centers appear cogent, there is some evidence that trauma outcomes do not suffer in states lacking such systems (9). Our findings showed no correlation between median crash-hospital-arrival time and the den- sity of trauma centers in a state (centers per million population). Since median time from EMS notification to arrival was less than $20 \%$ of total crash-to-hospital time, crash site factors or practices would seem to have played a major role in determining total transport times. Surprisingly, there was a significant correlation between mean arrival time and the density of all-level trauma centers in a state. This correlation was positive, implying longer crash to transport times in areas with higher trauma center densities. Despite statistical significance, this seemingly paradoxical effect of increasing trauma system coverage may have been due to chance alone. If, however, there was an increased prevalence of transport outliers (those cases in which hospital arrival occurred several hours after the crash) in states with the highest number of centers per population this might also explain a positive correlation. Such outliers were somewhat more likely to occur among rural crashes, and states with high ratios of all-level trauma centers to populations tended to be relatively rural. For example, the quite rural state of lowa has the highest number of trauma centers per million population of any state for which we had time data. There were 24 lowa crash victims (48\%) whose crash to hospital arrival times exceed the 46 minute mean. In Oklahoma, another predominantly rural state with a high density of trauma centers, $61 \%$ of victims arrived in hospital more than 46 minutes after their crash occurred.

This study is greatly limited by the absence of data on crash or arrival times for the majority of vehicle crashes in which children were victims. This absence was nearly universal in a number of states (for example, California - which reported full data on only two crashes). Furthermore, mortality after a crash, is affected by many more factors than are available in the FARS database. Among these are the quality of initial stabilization and the quality of definitive pediatric intensive care provided at the final receiving hospital. Finally, FARS is not designed as a research database, and there is no formal error checking for 
data entry. In consequence occasional very long delays in notification or transport time (rarely up to ten hours) may represent data entry errors rather than trauma system deficits. Within these limitations, the present study shows that there is a relatively long median time between U.S. crashes involving seriously injured children and their transport to an initial hospital. This transport time is somewhat longer for rural than for urban crashes, but while long delays in transport occur more commonly in rural areas, they are also seen in urban crashes. There is good evidence that, at least for adults, transport delays contribute to mortality.7 This study suggests that there may be opportunity to reduce both outlier delays and the shorten the overall time from crash to hospital arrival. Both of these outcomes are consistent with goals of Emergency Care for Children.(1) If transport delays could be reduced, pediatric intensive care units would have a better chance of ensuring quality survival for children critically injured in motor vehicle crashes.

\section{REFERENCES}

1. Committee on the future of emergency care in the United States health system. Emergency Care for Children: Growing Pains Washington: The National Academies Press;2006.

2. American Trauma Society. Trauma system agenda for the future. 2002. URL: http://www.nhtsa.dot.gov/people/injury/ems/TRAUMA SYSTEM/ as accessed 8/13/2006.

3. MacKenzie EJ, Hoyt DB, Sacra JC, Jurkovich, GJ, Carlini AR, Teitelbaum SD, Teter H. National inventory of hospital trauma centers. JAMA 2003;289:1515-1522.

4. Fatality Accident Reporting System. URL: http://www-fars.nhtsa.dot.gov/ as accessed 8/13/2006.

5. Briggs NC, Levine RS, Haliburton WP, Schlundt DG, Goldzweig I, Warren RC. The Fatality Analysis Reporting System as a tool for investigating racial and ethnic determinants of motor vehicle crash fatalities. Accid Anal Prev 2005;37(4):641-9.

6. Centers for Disease Control and Prevention (CDC). Child passenger deaths involving drinking drivers--United States, 1997-2002. MMWR Morb Mortal Wkly Rep 2004;53(4):77-9.

7. Harrington DT, Connolly M, Biffl WL, Majercik SD, Cioffi Wg. Transfer times to definitive care facilities are too long: a consequence of an immature trauma system. Ann Surg 2005;241:961-966.

8. Norwood S, Fernandez L, England J. The early effects of implementing American College of Surgeons level II criteria on transfer and survival rates at a rurally based community hospital. J Trauma 2002;39:240-244.

9. Rogers FB, Osler TM, Shackford SR, Cohen M, Camp I, Lesage M. Study of the outcome of patients transferred to a level I hospital after stabilization at an outlying hospital in a rural setting. J Trauma 1999;46:328-333. 